\title{
Insufficient Ablative Margin Determined by Early Computed Tomography May Predict the Recurrence of Hepatocellular Carcinoma after Radiofrequency Ablation
}

\author{
Wei Teng ${ }^{a} \quad$ Ka-Wai Liub ${ }^{b} \quad$ Chen-Chun Lin ${ }^{a} \quad$ Wen-Juei Jeng ${ }^{a}$ \\ Wei-Ting Chen ${ }^{a} \quad$ I-Shyan Sheen ${ }^{a}$ Chun-Yen Lin ${ }^{a}$ Shi-Ming Lina \\ aDivision of Hepatology, Liver Research Unit, Department of Gastroenterology and Hepatology, \\ bDepartment of Radiology, Chang Gung Memorial Hospital and Chang Gung University, College of \\ Medicine, Taipei, Taiwan (ROC)
}

\section{Key Words}

Early computed tomography - Hepatocellular carcinoma - Radiofrequency ablation .

Recurrence $\cdot$ Safety margin

\begin{abstract}
Tumor recurrence in hepatocellular carcinoma (HCC) patients after radiofrequency ablation (RFA) remains common; some studies have reported that insufficient ablative margin after RFA might contribute to HCC recurrence. The aim of this study was to investigate whether insufficient ablative safety margins determined by early computed tomography (CT) predicts $\mathrm{HCC}$ recurrence after RFA. This retrospective study recruited patients with a single HCC lesion after RFA in our department between May 2013 and March 2014. Early follow-up CT was performed within 7 days after RFA. An adequate ablative margin assessed by follow-up CT was defined as (maximum post-RFA CT radius) ${ }^{3} /(\text { maximum pre-RFA CT radius }+5 \mathrm{~mm})^{3}>1$. All patients in whom complete ablation was achieved underwent a CT scan every 3 months for early detection of HCC recurrence. In total, 72 patients (48 male, mean age 69.4 years) were analyzed. Of these, eight patients had local tumor progression, four had intra-hepatic distant recurrence, and two had extra-hepatic metastasis. Insufficient ablative margin, defined as an ablative volume with a safety margin of less than $5 \mathrm{~mm}$, was an important predictor of local tumor progression (LTP) $(p=0.015)$ and overall recurrence $(p=0.012)$. The sensitivity, speci-


ficity, and positive and negative predictive values of an insufficient ablative margin for predicting LTP and overall recurrence were $36.4 \%, 97.2 \%, 50.0 \%$, and $87.9 \%$, and $46.2 \%, 89.7 \%$, $42.9 \%$, and $87.9 \%$, respectively. An ablative volume with an ablative margin of less than $5 \mathrm{~mm}$ is associated with higher rates of both LTP and overall recurrence in HCC after RFA.

Copyright $(2015$ S. Karger AG, Basel

\section{Introduction}

Hepatocellular carcinoma (HCC) is the fifth most common cancer worldwide and the third most common cause of cancer mortality [1]. Radiofrequency ablation (RFA) has been accepted as an equivalent alternative to resection as an eradicative treatment for small HCC [2]. However, the local tumor progression (LTP) rate after RFA is higher than that after surgical resection [3, 4]. Several studies have indicated that the incidence of LTP after RFA ranges from $2 \%$ to $18 \%[5,6]$. LTP may occur because of untreated satellite lesions that are too small to detect on imaging prior to RFA [7] or because of insufficient ablation. Therefore, to avoid local recurrence in HCC treated with RFA, an ablative margin (AM) of at least $5 \mathrm{~mm}$ was suggested in one small population study $(n=85)$ [8].

In current practice, the technical success of RFA is determined by pre- and post-RFA enhanced computed tomography (CT) images [8-10]. Kim et al use prototype software with registration of post-RFA CT to pre-RFA CT images to assess the safety margin immediately after RFA [11]. However, the traditional approach of using two-dimensional section-by-section comparison of pre- and post-RFA CT images alone may be insufficient to assess the AM because of the three dimensional spatial structure of the ablation volume. Motoyama et al attempted to assess the utility of coronal reformations of 64-slice multi-detector computed tomography (64-MDCT) images in assessing the AM following RFA [12]. They found that evaluation of the AM on both horizontal and coronal or sagittal images was superior to that of horizontal images alone in terms of improved positive predictive values (PPVs) and negative predictive values (NPVs) for local recurrence. In the current study, we calculated the tumor volume to assess the AM following RFA and compared this method with the utility of two-dimensional section-by-section assessments of AM through horizontal alone or both horizontal and coronal reformations of dynamic CT. Furthermore, we also investigate whether early follow-up CT could predict recurrence in patients with HCC after RFA.

\section{Materials and Methods}

\section{Diagnosis of Hepatocellular Carcinoma}

Patients with a liver nodule detectable on ultrasonography underwent dynamic CT or magnetic resonance imaging (MRI) for a definitive diagnosis of HCC [13]. Arterial and portal phase dynamic CT images were obtained at approximately 35 and $70 \mathrm{~s}$, respectively, after injecting iodinated contrast material. Arterial and portal phase dynamic MR images were obtained at approximately 35 and $120 \mathrm{~s}$, respectively, after injecting gadolinium-diethylenetriamine pentaacetic acid (gadopentetate dimeglumine) or gadolinium-ethoxybenzyl-diethylenetriamine pentaacetic acid (Gd-EOB-DTPA [gadoxetate disodium]) [14]. Tumors with a typical enhancing pattern (i.e., hyperattenuation or hyperintensity during the arterial phase and hypoattenuation or hypointensity in the portal venous phase) were immediately diagnosed as HCCs. Percutaneous needle biopsy under ultrasound guidance was performed with an 18-gauge needle for the histologic diagnosis of hepatic nodules. Samples were fixed in $10 \%$ phosphate-buffered formalin, which was immediately followed by paraffin embedding and hematoxylin and eosin staining. An experienced liver pathologist examined the samples, and the diagnosis was made according to International Working Party criteria [15]. 


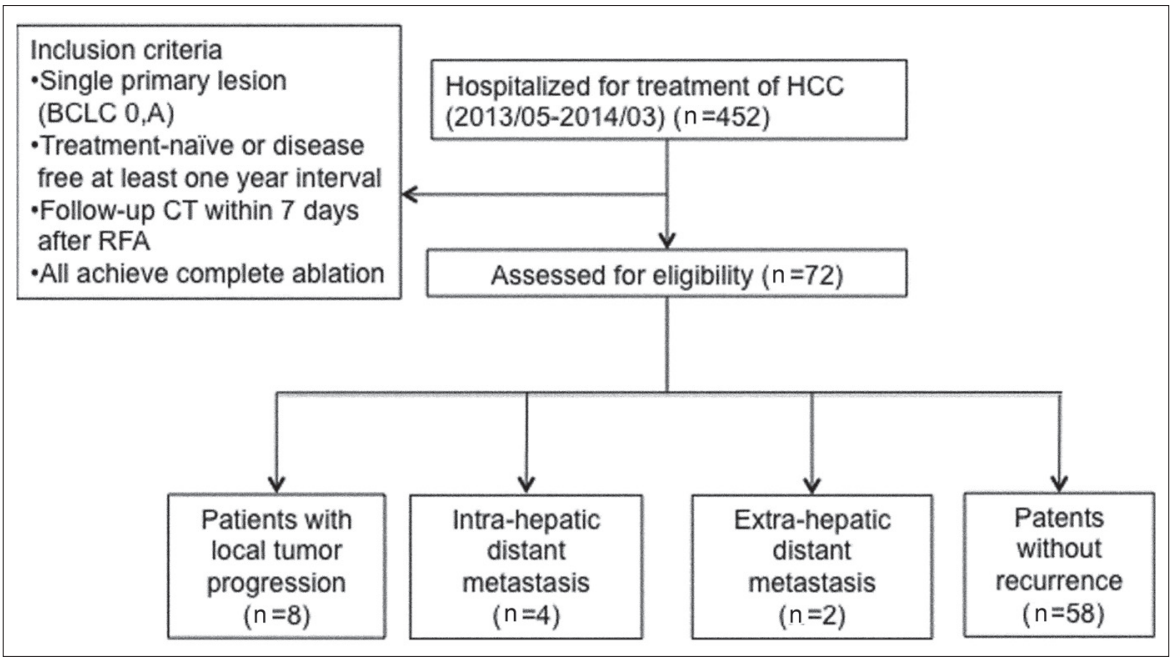

Fig. 1. Flow diagram showing the patients enrolled in this study.

\section{Study Design and Patient Population}

A total of 452 patients with HCC were treated with RFA in Linkou Chang-Gung Memorial Hospital between May 2013 and March 2014. Patients with a follow-up of at least 3 months after RFA were enrolled in this study. To evaluate the effects of RFA and to minimize bias, we enrolled only patients with a single lesion who were HCC treatment naive or HCC free for at least 1 year before HCC was diagnosed. A total of 72 HCC nodules were retrospectively analyzed in 72 patients treated with RFA (fig. 1).

\section{RFA Protocol}

Patients with portal vein tumor thrombosis, refractory ascites, or extrahepatic metastasis were excluded [16]. Briefly, RFA was performed percutaneously under ultrasound guidance. Straight electrodes with a 3- or 2-cm active tip were used. We often used a multiple overlapping ablation technique to create a larger ablation area. Artificial ascites or pleural effusion techniques $[17,18]$ were used when the target tumor was close to critical organs or located in the hepatic dome area. Radiofrequency energy was delivered for 9-12 min for each application. RFA was performed by three experienced hepatologists.

\section{CT Protocol}

CT examinations were performed less than 1 month before RFA by using a four-phase (pre-contrast, arterial, portal, delayed) technique with the MDCT system. The slice thickness was $5 \mathrm{~mm}$, and the table feed was $5 \mathrm{~mm}$. Each patient was injected with $100 \mathrm{ml}$ of a nonionic contrast material (iopamidol, Iopamiron 370; Bayer Yakuhin, Osaka, Japan) at a rate of $3.5 \mathrm{ml} / \mathrm{s}$ using a power injector (Nemoto Kyorindo, Tokyo, Japan). The bolus tracking method was used to scan each patient. The trigger threshold level was set at 200 Hounsfield units at the level of the descending aorta. The size of the nodule was determined on either the arterial or portal phase, depending on which phase could best depict the nodule. We assumed that tumors were a three-dimensional ellipsoid shape (fig. 2). We calculated the half maximum length of the tumor (labeled as "a") on the horizontal view, the half maximum height of the tumor (labeled as "b") on coronal or sagittal views, and the half maximum width of the tumor (labeled as "c") using horizontal views of CT or MRI images (fig. 3). The pre-RFA tumor volume was calculated as $4 / 3 \times \pi \times(a \times b \times c)$ under the assumption of ellipsoid shape. An adequate ablative margin assessed by follow-up CT was defined as

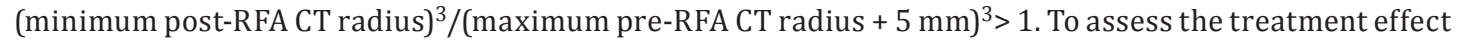
of RFA, early dynamic CT or MRI using 5-mm-slice scans was performed within 7 days after RFA. The absence of visible residuals on post-RFA CT or MR images of the arterial phase was confirmed for all nodules [19]. All patients underwent follow-up dynamic CT or MRI within 3 months after the last RFA procedure; these scans were repeated approximately every 3 months if LTP was not found. LTP was diagnosed when follow-up CT or MRI revealed new nodules that had developed in close proximity to a previous ablation zone. The assessments of safety margins (using both volumetric and two-dimensional section-by-section approaches) were performed retrospectively by one radiologist and two hepatologists. 


\section{Liver Cancer}

\begin{tabular}{l|l}
\hline Liver Cancer 2015;4:26-38 \\
\hline DOI: $10.1159 / 000343877$ & $\begin{array}{l}\text { C } 2015 \text { S. Karger AG, Basel } \\
\text { www.karger.com/lic }\end{array}$ \\
Publisned onlIne: February 17, 2015 &
\end{tabular}

Teng et al.: Insufficient Ablative Margin and Recurrence of HCC after RFA

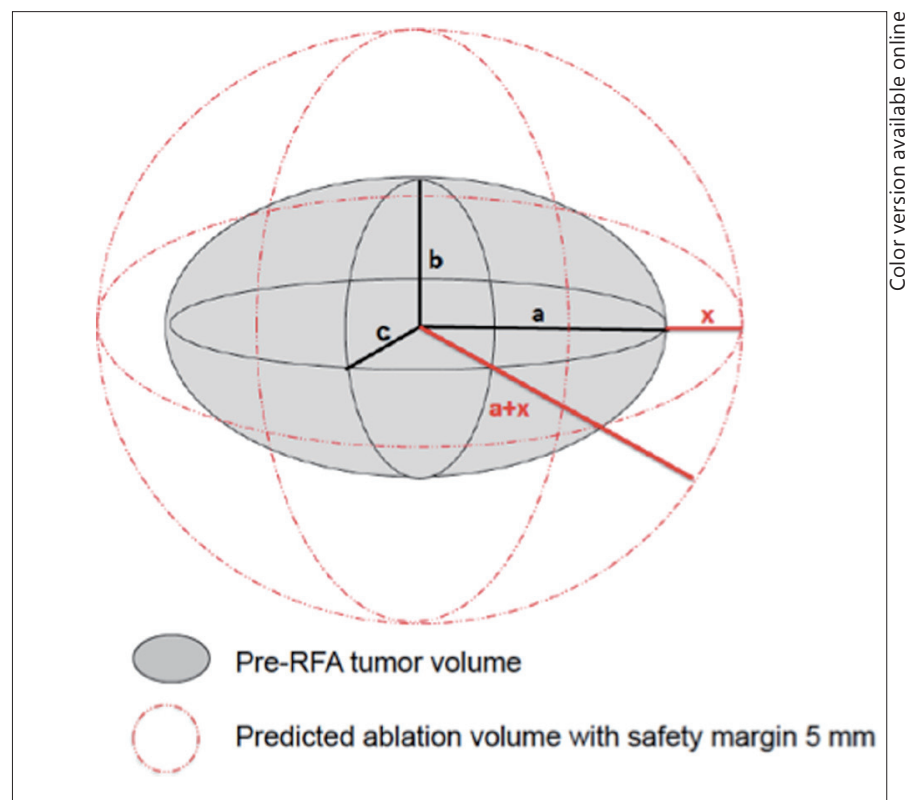

Fig. 2. Diagrammatic representation of the pre-RFA tumor volume and predicted ablation volume with an ablation margin of $5 \mathrm{~mm}$. The symbols $\mathrm{a}, \mathrm{b}, \mathrm{c}$, and $x$ represent half the tumor maximum length, width, and height and the ablation margin, respectively. An ablative volume with margin $5 \mathrm{~mm}$ covers the entire tumor.

\section{Statistical Analyses}

Statistical analyses were carried out by SPSS software, version 20.0 (SPSS, Chicago, IL, US). Descriptive data with a normal distribution are reported as mean \pm standard deviation (SD). Student's $t$ test and the Mann-Whitney U test were used to the assess differences between groups in normal-distributed and non-normal-distributed groups separately. The chi-squared test was used for categorical variables between two groups. Stepwise logistic regression analyses, both univariate and multivariate, were used to determine the correlation of the predictive factors to clinical outcomes. A two-tailed $p$ value $<0.05$ was considered to be statistically significant.

\section{Results}

Among the 72 patients participating in this study, 48 were male and the mean age was 69.4 years (range: 38.1-91.7 years) (table 1). Fifty-eight (80.6\%) patients were diagnosed with liver cirrhosis by imaging or liver biopsy. The underlying etiology was HBV or HCV infection $(n=62,86.1 \%)$, alcohol abuse $(n=4,5.6 \%)$, and cryptogenic etiology $(n=6,8.3 \%)$. The basic biochemistry data are listed in table 1 . An ablation volume with a 5 -mm safety margin was achieved in $59(81.9 \%)$ patients. The mean \pm SD tumor diameter, pre-RFA tumor volume, post-RFA actual ablation volume, estimated ablation volume with a safety margin of $5 \mathrm{~mm}$, and observation period were $23 \pm 10 \mathrm{~mm}, 7.6 \pm 9.8 \mathrm{~cm}^{3}, 44.1 \pm 35.8 \mathrm{~cm}^{3}, 24.8 \pm$ $24.0 \mathrm{~cm}^{3}$, and $221 \pm 83$ days respectively. No patients had major complications, such as internal bleeding, perforation, or sepsis after RFA.

Fourteen (19.4\%) patients encountered tumor recurrence, i.e., LTP $(n=8,11.1 \%)$, intrahepatic distant metastasis $(n=4,5.6 \%)$, and extra-hepatic metastasis $(n=2,2.8 \%)$ as diagnosed by follow-up dynamic CT scans. The mean disease-free interval after RFA was $153 \pm 69$ days (range, 44-259 days). 

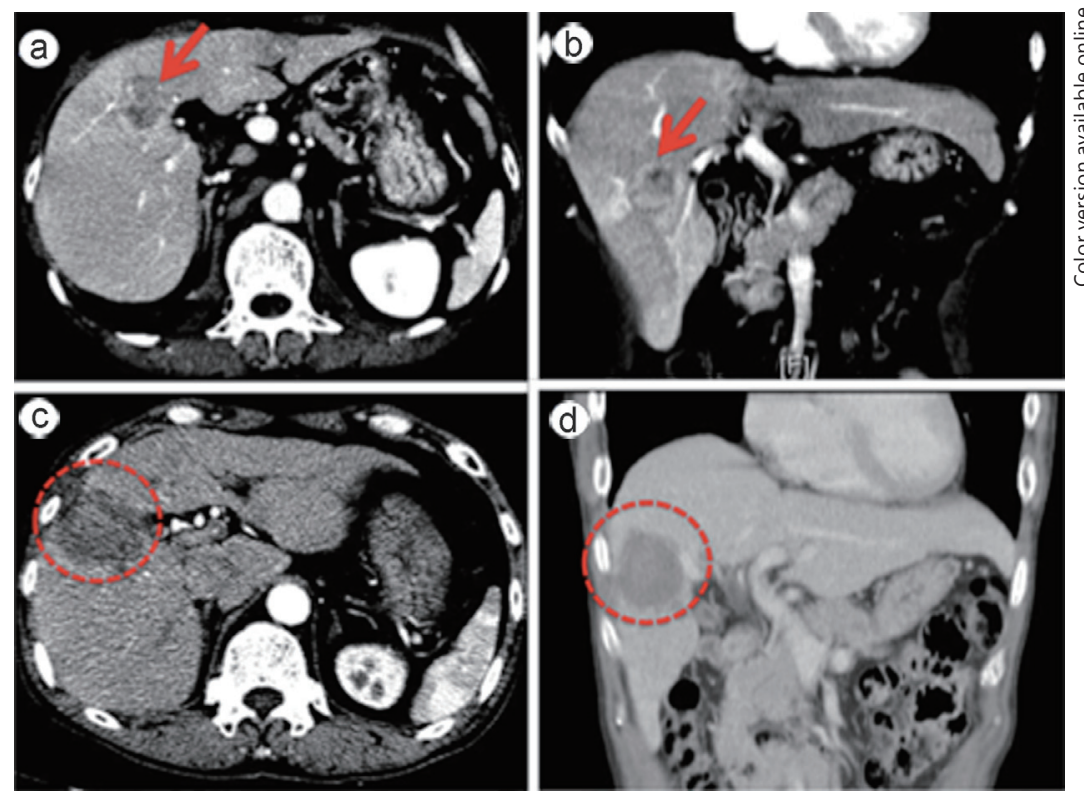

Fig. 3. Representative CT images of pre-RFA (a,b) and post-RFA (c,d). a An axial image of the tumor (arrow) in the hepatic arterial phase. b A coronal image of the tumor (arrow) in the hepatic arterial phase. c A post-RFA axial image. The tumor was evaluated as ablative margin (AM) sufficient (surrounded by the dotted line). d A post-RFA coronal image. The tumor was evaluated as AM sufficient (surrounded by the dotted line).

As shown in table 2, the significant differences between the LTP and non-LTP groups were the mean baseline alpha-fetoprotein (AFP) levels (1297 vs. $345 \mathrm{ng} / \mathrm{ml}, \mathrm{p}=0.049$ ), the use of artificial ascites or pleural effusion in high-risk locations (62.5\% vs. $27.6 \%, p=0.047)$, and the ablation volume being more than that for a safety margin of $5 \mathrm{~mm}(50.0 \% \mathrm{vs} .87 .9 \%$, $\mathrm{p}$ $=0.007$ ). The data for overall recurrence (including LTP, intra-hepatic distant metastasis and extra-hepatic metastasis) are shown in table 3. The significant differences between the overall recurrence and non-recurrence groups were mean baseline AFP levels (1075 vs. $345 \mathrm{ng} /$ $\mathrm{ml}, \mathrm{p}=0.049)$ and ablation volume being more than that for a safety margin of $5 \mathrm{~mm}(57.1 \%$ vs. $87.9 \%, p=0.015)$. Predictors for LTP and overall recurrence are shown in table 4 . Using univariate analysis, higher serum AFP level (OR =7.25, 95\%CI: 1.27-21.5, p = 0.030), use of artificial ascites or pleural effusion for high risk locations (OR $=4.38,95 \%$ CI: $1.40-20.47, p$ $=0.019)$, and ablation volume being more than that for a safety margin of $5 \mathrm{~mm}(\mathrm{OR}=0.16$, 95\%CI: $0.03-0.79, \mathrm{p}=0.024$ ) were found to be significant in LTP, whereas, for overall recurrence, higher serum AFP level (OR =4.15, 95\%CI: 1.33-17.29, $\mathrm{p}=0.016$ ) and ablation volume being more than that for a safety margin of $5 \mathrm{~mm}(\mathrm{OR}=0.163,95 \% \mathrm{CI}: 0.042-0.634, \mathrm{p}=0.009)$ were significant. By multivariate analysis, only ablation volume being more than that for a safety margin of $5 \mathrm{~mm}$ was found to be a significant predictor of LTP and overall recurrence, with odds ratios of 0.137 (95\%CI, 0.028-0.677; $p=0.015$ ) and 0.183 (95\%CI, 0.049-0.686; $p$ $=0.012$ ), respectively. For predicting LTP and overall recurrence, respectively, the evaluation of the sufficiency of the AM using both horizontal and coronal axes to calculate the volume rather than using the two-dimensional section-by-section approach on comparison of horizontal axes only had better sensitivity ( $36.4 \%$ vs. $23.3 \%, 46.2 \%$ vs. $30.3 \%$ ), specificity $(97.2 \%$ vs. $92.2 \%, 89.7 \%$ vs. $85.5 \%)$, negative predictive value (NPV) ( $87.9 \%$ vs. $60.3 \%, 87.9 \%$ vs. $60.3 \%$ ) and equal positive predictive value (PPV) (50.0\% vs. $50.0 \%$ and $42.9 \%$ vs. $42.9 \%$ ) (table 5). 
Table 1. Baseline characteristics of the 72 patients undergoing RFA for primary hepatocellular carcinoma

\begin{tabular}{|c|c|}
\hline Characteristic & Mean \pm SD (range) or number $(\%)$ \\
\hline Age (years) & $69.4 \pm 10.9(38.1-91.7)$ \\
\hline Gender (male, \%) & $48(66.7)$ \\
\hline Virus/alcoholic/cryptogenic (\%) & $62 / 4 / 6(86.1 / 5.6 / 8.3)$ \\
\hline Cirrhosis (\%) & $58(80.6)$ \\
\hline Chronic hepatitis/Child A/B/C & $14 / 45 / 12 / 1(19.4 / 62.5 / 16.7 / 1.4)$ \\
\hline Treatment naive (\%) & $59(81.9)$ \\
\hline Ascites (\%) & $9(12.5)$ \\
\hline INR & $1.2 \pm 0.1(0.9-1.6)$ \\
\hline Bilirubin total (mg/dl) & $1.0 \pm 0.9(0.2-6.7)$ \\
\hline Albumin (g/dl) & $3.68 \pm 0.60(2.38-4.69)$ \\
\hline $\operatorname{AFP}(\mathrm{ng} / \mathrm{ml})$ & $487.1 \pm 1562.5(1.4-9587.2)$ \\
\hline Creatinine (mg/dl) & $0.95 \pm 0.82(0.40-7.13)$ \\
\hline $\operatorname{ALT}(U / \mathrm{l})$ & $51 \pm 43(7-203)$ \\
\hline Platelet count (k) & $124 \pm 61(38-322)$ \\
\hline Tumor size (mm) & $23 \pm 10(8-49)$ \\
\hline Close to vessel (\%) & $25(34.7)$ \\
\hline Close to organ/subcapsular (\%) & $38(52.8)$ \\
\hline Use of artificial ascites/effusion (\%) & $22(30.6)$ \\
\hline Pre-RFA tumor volume $\left(\mathrm{cm}^{3}\right)$ & $7.6 \pm 9.8(0.2-47.7)$ \\
\hline $\begin{array}{l}\text { Estimated ablation volume with } \\
\text { safety margin of } 5 \mathrm{~mm}\left(\mathrm{~cm}^{3}\right)\end{array}$ & $24.8 \pm 24.0(3.1-107.5)$ \\
\hline Post-RFA ablation volume $\left(\mathrm{cm}^{3}\right)$ & $44.1 \pm 35.8(6.2-223.5)$ \\
\hline Follow up days & $221 \pm 83(102-392)$ \\
\hline Disease-free interval days & $153 \pm 69(44-259)$ \\
\hline
\end{tabular}

INR = international normalized ratio; AFP = alpha-fetoprotein; ALT = alanine aminotransferase; RFA = radiofrequency ablation.

\section{Discussion}

RFA is a curative treatment for small HCC lesions. An ablation volume encompassing both the target tumor and the surrounding liver parenchyma is mandatory to obtain a sufficient therapeutic response. Therefore, evaluating the AM is of paramount importance. The AM has been assessed using only axial enhanced CT and MRI images in previous articles; however, recent studies revealed that other modalities of diagnostic imaging such as super paramagnetic iron oxide (SPIO)-enhanced MRI and contrast-enhanced ultrasonography (CEUS) enabled a more precise evaluation of the AM [20, 21]. Imaging fusion techniques such as the registration of pre-RFA axial CT images onto post-RFA images appear to be useful and accurate for evaluating the AM $[22,23]$. However, because neither CEUS nor fusion techniques were available in our hospital, we use conventional dynamic CT or MRI to evaluate the safety margin after RFA, but we introduced the concept of three-dimensional spatial 
Table 2. Baseline characteristics of patients with LTP versus non-LTP of HCC after RFA

\begin{tabular}{|c|c|c|c|}
\hline Variables & $\begin{array}{l}\text { LTP } \\
(\mathrm{n}=8)\end{array}$ & $\begin{array}{l}\text { Non-LTP } \\
(\mathrm{n}=58)\end{array}$ & $\mathrm{p}$ value \\
\hline Age (years)* & $71.9 \pm 12.0(53.2-82.7)$ & $69.8 \pm 10.8(38.1-91.7)$ & 0.404 \\
\hline Gender (male,\%) & $5(62.5)$ & $38(65.5)$ & 0.867 \\
\hline Virus/alcoholic/cryptogenic (\%) & $8 / 0 / 0(100 / 0 / 0)$ & $48 / 4 / 6(82.8 / 6.9 / 10.3)$ & 0.719 \\
\hline Cirrhosis (\%) & $7(87.5)$ & $46(79.3)$ & 0.688 \\
\hline Chronic hepatitis/Child A/B/C & $\begin{array}{l}1 / 7 / 0 / 0 \\
(12.5 / 87.5 / 0 / 0)\end{array}$ & $\begin{array}{l}12 / 34 / 11 / 1 \\
(20.7 / 58.6 / 19.0 / 1.7)\end{array}$ & 0.448 \\
\hline Treatment naive (\%) & $6(75.0)$ & $48(82.8)$ & 0.630 \\
\hline PT/INR* & $1.1 \pm 0.1(1.0-1.2)$ & $1.2 \pm 0.1(0.9-1.5)$ & 0.054 \\
\hline Bilirubin total (mg/dl)* & $0.75 \pm 0.41(0.4-1.7)$ & $0.97 \pm 0.70(0.2-3.5)$ & 0.385 \\
\hline Albumin (g/dl)* & $\begin{array}{l}3.86 \pm 0.48 \\
(3.30-4.60)\end{array}$ & $3.64 \pm 0.61(2.38-4.61)$ & 0.556 \\
\hline $\operatorname{AFP}(\mathrm{ng} / \mathrm{ml})^{*}$ & $1297 \pm 3354(4-9587)$ & $345 \pm 1152(1-5270)$ & 0.049 \\
\hline Creatinine $(\mathrm{mg} / \mathrm{dl})^{*}$ & $\begin{array}{l}0.93 \pm 0.24 \\
(0.54-1.21)\end{array}$ & $0.97 \pm 0.90(0.4-7.13)$ & 0.256 \\
\hline $\operatorname{ALT}(\mathrm{U} / \mathrm{l})^{*}$ & $45 \pm 42(14-144)$ & $54 \pm 45(10-203)$ & 0.401 \\
\hline Platelet count (k)* & $120 \pm 68(52-263)$ & $124 \pm 60(38-322)$ & 0.791 \\
\hline Tumor size (mm)* & $28 \pm 12(19-48)$ & $23 \pm 10(8-49)$ & 0.143 \\
\hline Close to vessel (\%) & $4(50.0)$ & $20(34.5)$ & 0.448 \\
\hline Close to subcapsular/organ (\%) & $4(50.0)$ & $30(51.7)$ & 0.927 \\
\hline Use of artificial ascites/effusion (\%) & $5(62.5)$ & $16(27.6)$ & 0.047 \\
\hline $\begin{array}{l}\text { Estimated ablation volume with } \\
\text { safety margin of } 5 \mathrm{~mm}\left(\mathrm{~cm}^{3}\right)\end{array}$ & $37.6 \pm 38.5(12.8-102.2)$ & $24.0 \pm 22.2(3.1-107.5)$ & 0.140 \\
\hline Post-RFA ablation volume $\left(\mathrm{cm}^{3}\right)$ & $\begin{array}{l}37.8 \pm 19.7 \\
(16.0-68.4)\end{array}$ & $46.2 \pm 38.9(6.2-223.5)$ & 0.937 \\
\hline $\begin{array}{l}\text { Ablation volume } \geq \text { safety margin of } \\
5 \mathrm{~mm}(\%)\end{array}$ & $4(50.0)$ & $51(87.9)$ & 0.007 \\
\hline
\end{tabular}

$\mathrm{LTP}=$ local tumor progression; $\mathrm{PT}=$ prothrombin time; INR = international normalized ratio; $\mathrm{AFP}=$ alpha-fetoprotein; ALT = alanine aminotransferase; RFA = radiofrequency ablation. ${ }^{*}$ Given as mean \pm SD (range).

structure through calculating the ablation volume encompassing both the target tumor and the surrounding liver parenchyma. Coronal or sagittal images can demonstrate the vascular and biliary systems in the liver in the longitudinal axis and provide additional information on the location and extent of HCC. Coronal reformatting techniques can also recognize the relationship between tumor and hepatic vasculatures, which is essential for the accurate preoperative planning of RFA [12]. We analyzed LTP of HCC after percutaneous RFA independently from other recurrence, e.g., intra-hepatic distant metastasis and extra-hepatic metastasis, because each type of recurrence has a specific mechanism of pathogenesis. Generally, LTP was considered related to residual tumor cells that have spread microscopically beyond the ablative margin, although there is a possibility of de novo occurrence at that site. Therefore, LTP may be more closely associated with a treatment methodology or result, the local environment of the tumor (such as contact with vessels), and the characteristics of the tumor 
Table 3. Baseline characteristics of patients with overall recurrence and non-recurrence of HCC after RFA

\begin{tabular}{|c|c|c|c|}
\hline Variables & $\begin{array}{l}\text { Overall recurrence } \\
(n=14)\end{array}$ & $\begin{array}{l}\text { Non-recurrence } \\
(n=58)\end{array}$ & $\mathrm{p}$ value \\
\hline Age (years)* & $68.0 \pm 11.4(51.5-82.7)$ & $70.0 \pm 10.8(38.1-91.7)$ & 0.629 \\
\hline Gender (male,\%) & $10(71.4)$ & $38(65.5)$ & 0.761 \\
\hline Virus/alcoholic/cryptogenic (\%) & $14 / 0 / 0(100 / 0 / 0)$ & $48 / 4 / 6(82.7 / 5.8 / 11.5)$ & 0.208 \\
\hline Cirrhosis (\%) & $12(85.7)$ & $46(79.3)$ & 0.723 \\
\hline Chronic hepatitis/Child A/B/C & $\begin{array}{l}2 / 11 / 1 / 0 \\
(14.3 / 78.6 / 7.1 / 0)\end{array}$ & $\begin{array}{l}12 / 34 / 11 / 1 \\
(20.7 / 58.6 / 19.0 / 1.7)\end{array}$ & 0.519 \\
\hline Treatment naive (\%) & $11(78.6)$ & $48(82.8)$ & 0.715 \\
\hline PT/INR* & $1.1 \pm 0.2(1.0-1.6)$ & $1.2 \pm 0.1(0.9-1.5)$ & 0.112 \\
\hline Bilirubin total (mg/dl)* & $1.1 \pm 1.6(0.3-6.7)$ & $1.0 \pm 0.7(0.2-3.5)$ & 0.247 \\
\hline Albumin (g/dl)* & $3.86 \pm 0.53(3.03-4.69)$ & $3.64 \pm 0.61(2.38-4.69)$ & 0.370 \\
\hline $\operatorname{AFP}(\mathrm{ng} / \mathrm{ml})^{*}$ & $1075 \pm 2656(1.8-9587.2)$ & $345 \pm 1152(1.4-5270.3)$ & 0.049 \\
\hline Creatinine (mg/dl)* & $0.90 \pm 0.33(0.46-1.73)$ & $0.97 \pm 0.90(0.40-7.13)$ & 0.490 \\
\hline $\operatorname{ALT}(U / \mathrm{l})^{*}$ & $39 \pm 33(7-144)$ & $54 \pm 45(10-203)$ & 0.179 \\
\hline Platelet count $(\mathrm{k})^{*}$ & $123 \pm 63(47-263)$ & $124 \pm 60(38-322)$ & 0.892 \\
\hline Tumor size $(\mathrm{mm})^{*}$ & $25 \pm 11(14-48)$ & $23 \pm 10(8-49)$ & 0.564 \\
\hline Close to vessel (\%) & 5 (35.7) & $20(34.5)$ & 0.931 \\
\hline Close to organ/subcapsular (\%) & $8(57.1)$ & 30 (51.7) & 0.773 \\
\hline Use of artificial ascites/effusion (\%) & $6(42.9)$ & $16(27.6)$ & 0.335 \\
\hline $\begin{array}{l}\text { Estimated ablation volume with } \\
\text { safety margin of } 5 \mathrm{~mm}\left(\mathrm{~cm}^{3}\right)\end{array}$ & $28.4 \pm 31.2(7.2-102.2)$ & $24.0 \pm 22.2(3.0-107.5)$ & 0.564 \\
\hline Post-RFA ablation volume $\left(\mathrm{cm}^{3}\right)$ & $35.6 \pm 15.8(16.0-68.4)$ & $46.2 \pm 38.9(6.2-223.5)$ & 0.765 \\
\hline $\begin{array}{l}\text { Ablation volume } \geq \text { safety margin } \\
\text { of } 5 \mathrm{~mm}(\%)\end{array}$ & $8(57.1)$ & $51(87.9)$ & 0.015 \\
\hline
\end{tabular}

LTP = local tumor progression; PT = prothrombin time; INR = international normalized ratio; AFP = alpha-fetoprotein; $\mathrm{ALT}=$ alanine aminotransferase; $\mathrm{RFA}=$ radiofrequency ablation. ${ }^{*}$ Given as mean $\pm \mathrm{SD}$ (range).

itself rather than the systemic condition of the patient. How to evaluate an accurate ablation margin plays an important role in such groups of patients. 64-MDCT is currently widely used because of its low cost, short examination time, and wide availability. Coronal or sagittal reformatted CT images obtained from 64-MDCT have also been shown to be particularly useful in both the detection of tumors and tumor progression in various cancers, including HCC. Tomonari et al [23] found that the site of recurrence was most commonly observed in the craniocaudal direction. Motoyama et al [12] used this concept and found that the assessment ability of the AM using the two-dimensional section-by-section approach to compare preand post-ablation lesion size based on both horizontal and coronal images was better than that based on horizontal images only, including better sensitivity, specificity, PPV and NPV. In our study, we also found the same satisfactory results. We calculated the tumor size through the ablation zone in three spatial axes from horizontal and coronal/sagittal views by using pre/post-RFA CT images. We found that if the ablation zone in each of the three spatial axes could achieve a minimum safety margin of $5 \mathrm{~mm}$, patients will have a lower LTP $(2.8 \% \mathrm{vs}$. 

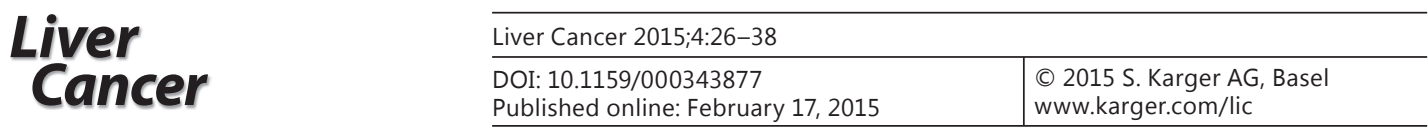

Teng et al.: Insufficient Ablative Margin and Recurrence of HCC after RFA

Table 4. Logistic regression of risk factors associated with local tumor progression and overall recurrence

\begin{tabular}{|c|c|c|c|c|c|c|}
\hline \multirow[t]{2}{*}{ Predictor variables } & \multicolumn{3}{|c|}{ Univariate } & \multicolumn{3}{|c|}{ Multivariate } \\
\hline & $\mathrm{OR}$ & $95 \% \mathrm{CI}$ & $\mathrm{p}$ value & OR & $95 \% \mathrm{CI}$ & $\mathrm{p}$ value \\
\hline \multicolumn{7}{|c|}{ Local tumor progression } \\
\hline AFP level & 7.25 & $1.27-21.5$ & 0.030 & & & \\
\hline \multicolumn{7}{|c|}{ Use of artificial ascites or pleural effusion } \\
\hline No & 1 & & & & & \\
\hline Yes & 4.38 & $1.40-20.47$ & 0.019 & & & \\
\hline \multicolumn{7}{|c|}{ Ablation volume $\geq$ safety margin of $5 \mathrm{~mm}$} \\
\hline No & 1 & & & 1 & & \\
\hline Yes & 0.16 & $0.03-0.79$ & 0.024 & 0.137 & $0.028-0.677$ & 0.015 \\
\hline \multicolumn{7}{|l|}{ Overall recurrence } \\
\hline AFP level & 4.15 & $1.33-17.29$ & 0.016 & & & \\
\hline \multicolumn{7}{|c|}{ Ablation volume $\geq$ safety margin of $5 \mathrm{~mm}$} \\
\hline No & 1 & & & 1 & & \\
\hline Yes & 0.163 & $0.042-0.634$ & 0.009 & 0.183 & $0.049-0.686$ & 0.012 \\
\hline
\end{tabular}

$23.3 \%, \mathrm{p}=0.019)$ and a lower overall recurrence rate $(10.3 \%$ vs. $30.3 \%, \mathrm{p}=0.040)$. The evaluation of AM sufficiency with both horizontal and coronal axes instead of with horizontal axes alone resulted in better sensitivity ( $26.7 \%$ vs. $23.3 \%, 35.3 \%$ vs. $30.3 \%)$, specificity $(92.7 \%$ vs. $92.2 \%, 86.4 \%$ vs. $85.5 \%$ ), positive predictive value (PPV) ( $87.5 \%$ vs. $50.0 \%, 71.4 \%$ vs. $42.9 \%$ ), and negative predictive value (NPV) ( $81.0 \%$ vs. $60.3 \%, 81.0 \%$ vs. $60.3 \%$ ) for the prediction of LTP and overall recurrence, respectively (table 5).

Because a tumor is a three-dimensional spatial structure with an irregular shape, it is not surprising that we could not adequately assess the safety ablation margin using only a conventional two-dimensional section-by-section comparison of pre- and post-RFA CT images. We assumed that tumors are approximately ellipsoid in shape and calculated the volume as $4 / 3 \times \pi \times(a \times b \times c)$ where $\mathrm{a}$, b, and $\mathrm{c}$ are half the tumor maximum length, width, and height respectively. Because cellular proliferation and resistance to apoptosis are significantly increased after incomplete thermal ablation [24], we try to create a large ablation volume with a safety margin of $5 \mathrm{~mm}$, i.e., $4 / 3 \times \pi \times(\text { maximum pre-RFA CT radius }+5 \mathrm{~mm})^{3}$, to cover the whole tumor. Most of our patients ( $\mathrm{n}=59,81.9 \%$ ) could achieve an ablation zone corresponding to a relative low LTP $(7.2 \%$ vs. $36.4 \%, p=0.007)$ and overall recurrence $(13.6 \%$ vs. $46.2 \%, p=0.015$ ) rate. As expected, use of the above mentioned ablated volume to predict local progression and overall recurrence had better sensitivity and specificity than not only use of conventional horizontal axes but also use of horizontal plus coronal axes in our study (table 5). However, it is difficult to develop an ablative margin of at least $5 \mathrm{~mm}$ in the management of HCC in high-risk locations such as contiguous vessels, subcapsular regions, or near vital organs. It has been suggested that the presence of blood vessels contiguous to HCC affects LTP after RFA because of the heat sink effect [8]. However, in our study, the presence of contiguous vessels was not a significant factor $(p=0.448$ and 0.931 for LTP and overall recurrence, respectively), possibly because most of the contiguous vessels were hepatic veins rather than portal veins. In fact, Kim et al. [25] reported that aggressive ablation of the portion of the tumor close to a hepatic vein might be useful to prevent LTP. Besides, we could solve these problems by combining use of ethanol injection and using artificial ascites 


\section{Liver

Table 5. Sensitivity, specificity, positive predictive value, and negative predictive value of an insufficient ablation margin $(<5 \mathrm{~mm})$ assessed in three different ways for predicting local tumor progression and overall recurrence

Local tumor progression

$(\%)$

Sensitivity

Only horizontal axes (2D, S/S)

Both horizontal and coronal axes (2D, S/S)

Volume from horizontal and coronal images

Specificity

Only horizontal axes (2D, S/S)

Both horizontal and coronal axes (2D, S/S)

Volume from horizontal and coronal images

Positive predictive value

Only horizontal axes (2D, S/S)

Both horizontal and coronal axes (2D, S/S)

Volume from horizontal and coronal images

Negative predictive value

Only horizontal axes (2D, S/S)

Both horizontal and coronal axes (2D, S/S)

Volume from horizontal and coronal images

23.3

26.7

36.4

92.2

92.7

97.2

50.0

87.5

50.0
30.3

46.2

85.5

86.4

89.7

71.4

2D, S/S = Two-dimensional section-by-section assessment of whether a 5-mm safety margin was achieved.

or pleural effusion to achieve complete ablation [26]. In our study, use of artificial ascites or pleural effusion and adequate ablation margin for high-risk locations indeed decreased the LTP rate $(13.3 \%$ vs. $50.0 \%, \mathrm{p}=0.043)$ and overall recurrence rate $(18.8 \%$ vs. $50.0 \%$, $\mathrm{p}=0.048$ ) (fig. 4A, 4B).

There are some limitations in the present study. First, it was conducted in a retrospective cohort with a small sample size. To reduce the effects of other factors, we focused on patients who had primary single small HCC that was completely ablated. These results must be confirmed in larger prospective studies. Second, the short follow-up period may underestimate the likelihood of LTP or recurrence. A longer follow-up time is required to validate our findings. Third, other modalities such as contrast-enhanced ultrasonography (CEUS) can be used to assess the efficacy of RFA for HCC, with the potential to reduce the number of CT scans required for confirmation in patients with impaired renal function [21]; however, CEUS was not available in this study setting.

In conclusion, an ablation volume with an ablation margin greater than $5 \mathrm{~mm}$ is a significant factor contributing to lower LTP and overall recurrence in HCC after RFA. Further studies with larger populations are required to validate our findings. 


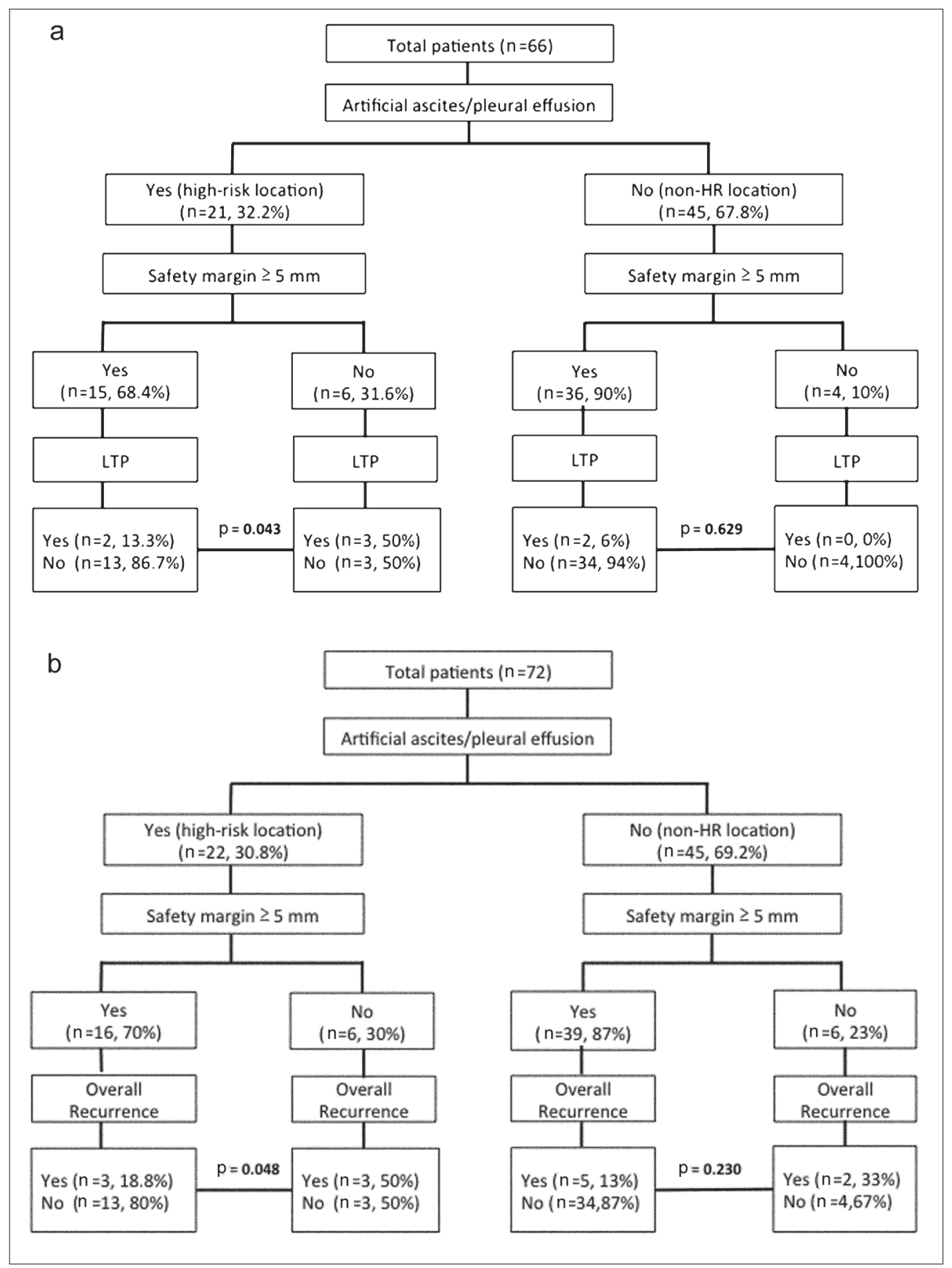

Fig. 4. a Flow diagram showing how the use of artificial ascites or pleural effusion for high risk locations and a safety margin of $5 \mathrm{~mm}$ affect local tumor progression (LTP). Patients with intra-hepatic distant metastasis $(n=4)$ and extra-hepatic metastasis $(n=2)$ were excluded. b Flow diagram showing how the use of artificial ascites or pleural effusion for high risk locations and a safety margin of $5 \mathrm{~mm}$ affect overall recurrence including LTP ( $\mathrm{n}=8)$, intra-hepatic distant metastasis $(\mathrm{n}=4)$ and extra-hepatic metastasis $(\mathrm{n}=2)$. 


\section{References}

1 El-Serag HB, Rudolph KL: Hepatocellular carcinoma: epidemiology and molecular carcinogenesis. Gastroenterology 2007;132:2557-2576.

-2 Kim YS, Lim HK, Rhim H, Lee MW, Choi D, Lee WJ, Paik SW, Koh KC, Lee JH, Choi MS, Gwak GY, Yoo BC: Ten-year outcomes of percutaneous radiofrequency ablation as first-line therapy of early hepatocellular carcinoma: analysis of prognostic factors. J Hepatol 2013;58:89-97.

3 Huang J, Yan L, Cheng Z, Wu H, Du L, Wang J, Xu Y, Zeng Y: A randomized trial comparing radiofrequency ablation and surgical resection for HCC conforming to the Milan criteria. Ann Surg 2010;252:903-912.

4 Mise Y, Sakamoto Y, Ishizawa T, Kaneko J, Aoki T, Hasegawa K, Sugawara Y, Kokudo N: A worldwide survey of the current daily practice in liver surgery. Liver Cancer 2013;2:55-66.

5 Livraghi T, Meloni F, Di Stasi M, Rolle E, Solbiati L, Tinelli C, Rossi S: Sustained complete response and complications rates after radiofrequency ablation of very early hepatocellular carcinoma in cirrhosis: Is resection still the treatment of choice? Hepatology 2008;47:82-89.

-6 Yan K, Chen MH, Yang W, Wang YB, Gao W, Hao CY, Xing BC, Huang XF: Radiofrequency ablation of hepatocellular carcinoma: long-term outcome and prognostic factors. Eur J Radiol 2008;67:336-347.

7 Okusaka T, Okada S, Ueno H, Ikeda M, Shimada K, Yamamoto J, Kosuge T, Yamasaki S, Fukushima N, Sakamoto M: Satellite lesions in patients with small hepatocellular carcinoma with reference to clinicopathologic features. Cancer 2002;95:1931-1937.

8 Nakazawa T, Kokubu S, Shibuya A, Ono K, Watanabe M, Hidaka H, Tsuchihashi T, Saigenji K: Radiofrequency ablation of hepatocellular carcinoma: correlation between local tumor progression after ablation and ablative margin. AJR Am J Roentgenol 2007;188:480-488.

-9 Barker DW, Zagoria RJ, Morton KA, Kavanagh PV, Shen P: Evaluation of liver metastases after radiofrequency ablation: utility of 18F-FDG PET and PET/CT. AJR Am J Roentgenol 2005;184:1096-1102.

$\checkmark 10$ Schraml C, Clasen S, Schwenzer NF, Koenigsrainer I, Herberts T, Claussen CD, Pereira PL: Diagnostic performance of contrast-enhanced computed tomography in the immediate assessment of radiofrequency ablation success in colorectal liver metastases. Abdom Imaging 2008;33:643-651.

$\checkmark 11$ Kim KW, Lee JM, Klotz E, Kim SJ, Kim SH, Kim JY, Han JK, Choi BI: Safety margin assessment after radiofrequency ablation of the liver using registration of preprocedure and postprocedure CT images. AJR Am J Roentgenol 2011;196:W565-72.

12 Motoyama T, Ogasawara S, Chiba T, Higashide T, Yokota H, Kanogawa N, Suzuki E, Ooka Y, Tawada A, Irie R, Ochi S, Masuda Y, Uno T, Yokosuka O: Coronal reformatted CT images contribute to the precise evaluation of the radiofrequency ablative margin for hepatocellular carcinoma. Abdom Imaging 2014;39:262-268.

13 Murakami T, Tsurusaki M: Hy pervascular benign and malignant liver tumors that require differentiation from hepatocellular carcinoma: key points of imaging diagnosis. Liver Cancer 2014;3:85-96.

14 Ichikawa T, Sano K, Morisaka H: Diagnosis of pathologically early HCC with EOB-MRI: experiences and current consensus. Liver Cancer 2014;3:97-107.

15 International Working Party: Terminology of nodular hepatocellular lesions. Hepatology 1995;22:983993.

16 Tateishi R, Shiina S, Teratani T, Obi S, Sato S, Koike Y, Fujishima T, Yoshida H, Kawabe T, Omata M: Percutaneous radiofrequency ablation for hepatocellular carcinoma. An analysis of 1000 cases. Cancer 2005;103:1201-1209.

17 Kondo Y, Yoshida H, Shiina S, Tateishi R, Teratani T, Omata M: Artificial ascites technique for percutaneous radiofrequency ablation of liver cancer adjacent to the gastrointestinal tract. Br J Surg 2006;93:12771282.

18 Uehara T, Hirooka M, Ishida K, Hiraoka A, Kumagi T, Kisaka Y, Hiasa Y, Onji M: Percutaneous ultrasoundguided radiofrequency ablation of hepatocellular carcinoma with artificially induced pleural effusion and ascites. J Gastroenterol 2007;42:306-311.

19 Ahmed M, Solbiati L, Brace CL, Breen DJ, Callstrom MR, Charboneau JW, Chen MH, Choi BI, de Baère T, Dodd GD 3rd, Dupuy DE, Gervais DA, Gianfelice D, Gillams AR, Lee FT Jr, Leen E, Lencioni R, Littrup PJ, Livraghi T, Lu DS, McGahan JP, Meloni MF, Nikolic B, Pereira PL, Liang P, Rhim H, Rose SC, Salem R, Sofocleous CT, Solomon SB, Soulen MC, Tanaka M, Vogl TJ, Wood BJ, Goldberg SN, International Working Group on Image-guided Tumor Ablation Interventional Oncology Sans Frontières Expert Panel Technology Assessment Committee of the Society of Interventional Radiology Standard of Practice Committee of the Cardiovascular and Interventional Radiological Society of Europe: Image-guided tumor ablation: standardization of terminology and reporting criteria-a 10-year update. Radiology 2014;273:241-260.

20 Tokunaga S, Koda M, Matono T, Sugihara T, Nagahara T, Ueki M, Murawaki Y, Kakite S, Yamashita E: Assessment of ablative margin by MRI with ferucarbotran in radiofrequency ablation for liver cancer: comparison with enhanced CT. Br J Radiol 2012;85:745-752.

21 Inoue T, Kudo M, Hatanaka K, Arizumi T, Takita M, Kitai S, Yada N, Hagiwara S, Minami Y, Sakurai T, Ueshima K, Nishida N: Usefulness of contrast-enhanced ultrasonography to evaluate the post-treatment responses of radiofrequency ablation for hepatocellular carcinoma: comparison with dy namic CT. Oncology 2013;84(Suppl 1):51-57.

-22 Shin S, Lee JM, Kim KW, Joo I, Han JK, Choi BI, Klotz E: Postablation assessment using follow-up registration of CT images before and after radiofrequency ablation (RFA): prospective evaluation of midterm therapeutic results of RFA for hepatocellular carcinoma. AJR Am J Roentgenol 2014;203:70-77. 
-23 Tomonari A, Tsuji K, Yamazaki H, Aoki H, Kang JH, Kodama Y, Sakurai Y, Maguchi H: Feasibility of fused imaging for the evaluation of radiofrequency ablative margin for hepatocellular carcinoma. Hepatology 2013; 43:728-734.

24 Li Y, Brown RE, Martin RC: Incomplete thermal ablation of hepatocellular carcinoma: effects on tumor proliferation. J Surg Res 2013;181:250-255.

25 Kim YS, Lee WJ, Rhim H, Lim HK, Choi D, Lee JY: The minimal ablative margin of radiofrequency ablation of hepatocellular carcinoma ( $>2$ and $<5 \mathrm{~cm}$ ) needed to prevent local tumor progression: 3D quantitative assessment using CT image fusion. AJR Am J Roentgenol 2010;195:758-765.

-26 Lin SM: Local ablation for hepatocellular carcinoma in taiwan. Liver Cancer 2013;2:73-83. 